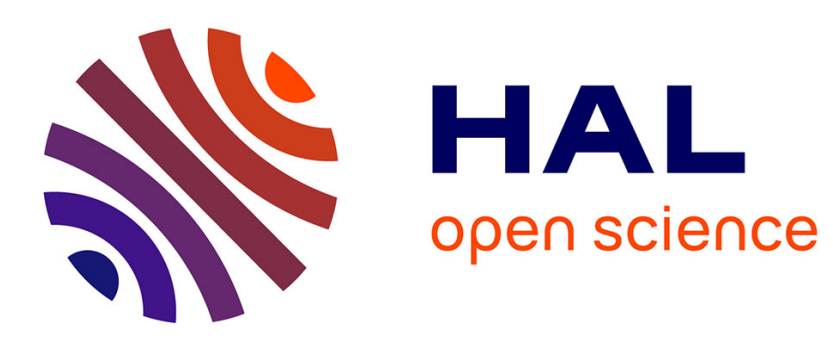

\title{
Violence et ordre social dans une société amazonienne Les Yanomami du Venezuela
}

Catherine Alès

\section{To cite this version:}

Catherine Alès. Violence et ordre social dans une société amazonienne Les Yanomami du Venezuela. Études rurales, 1984. halshs-02546346

\section{HAL Id: halshs-02546346 \\ https://shs.hal.science/halshs-02546346}

Submitted on 17 Apr 2020

HAL is a multi-disciplinary open access archive for the deposit and dissemination of scientific research documents, whether they are published or not. The documents may come from teaching and research institutions in France or abroad, or from public or private research centers.
L'archive ouverte pluridisciplinaire HAL, est destinée au dépôt et à la diffusion de documents scientifiques de niveau recherche, publiés ou non, émanant des établissements d'enseignement et de recherche français ou étrangers, des laboratoires publics ou privés. 


\section{Violence et ordre social dans une société amazonienne}

\section{Les Yanomami du Venezuela}

Après le premier témoignage recueilli de la bouche d'Héléna Valero par E. Biocca [1965], c'est avec les travaux de N. A. Chagnon $[1966,1968$ a 1968b, 1968c) qui contiennent des descriptions et des analyses essentielles ${ }^{1}$, que la guere et la violence chez les Yanomami vont ètre connues et tenir une place de choix dans la littérature anthropologique, quoique utilisées parfois de manière réduc trice.

Le phénomène guerrier propre à cette société ne peut, en effet, être assimilè à des rapports dhostilité qu'entretiendraient des groupes en competition pour l'usage de territoires ${ }^{2}$, l'obtention de biens ou de femmes. Certes les groupes yanomami combattent et inscrivent leur violence dans l'espace, afin d'assurer leur sécurité et d'affirmer leur souveraineté dans des limites territoriales. Mais, dans la plupart des cas, la guerre yanomami trouve sa genèse dans des conflits internes, à l'occasion desquels, la violence s'alimentant d'elle-mème, se développe une logique d'affrontement constitutive de l'ordre social et révélatrice de sa structure.

\section{ETHQUE ET PRATIQUES DE LA VIOLENCE CHEZ LES YANOMAMI}

Les Yanomami forment un sous-groupe dialectal d'environ 10000 personnes, le plus grand de la famille Yanoama (20 000 personnes) dont le territoire s'étend de part et d'autre de la frontière séparant le Venezuela et le Brésil.

Leur modèle résidentiel et territorial reflète les différents types de rapports socio-politiques quils entretiennent entre eux. Ils vivent en petites communautés semiendogames d'une soixantaine d'individus en moyenne, groupes de parents et d'alliés composés idéalement selon le modèle de l'èchange des sceurs. Ces communautés résidentielles sont les seules unités reconnues et nommées par les Yanomami. Sur le pourtour de l'espace circulaire dhabitation shapono, un ou plusieurs segments de parents s'ordonnent successivement, chacun comprenant une série de foyers domestiques. Dans les communautés de grande taille, on peut observer l'existence de bandes distinctes guidées en général par un ainé. Ce niveau d'organisation sociale est repérable dans la distribution de l'espace résidentiel et cultivé et surtout lors des périodes de nomadisme au moment où, éventuellement, ils se séparent pour sinstaller dans des campements provisoires en forêt ${ }^{3}$. 
On observe encore que quelques maisons communes, trois ou quatre le plus souvent, distantes les unes des autres de quelques kilomètres seulement et parfois plus étroitement regroupées, forment une sorte d'association. Leur proximité permet un contact et une communication permanents ${ }^{4}$. Elles sont unies socialement entre elles de manière privilégiée par l'échange matrimonial et cérémoniel et par la solidarité militaire - sans que soit pour autant garantie l'absence dhos. tilités entre elles -, et elles développent un consensus concernant l'utilisation des ressources végétales et animales comprises sur un territoire étendu qu'elles sont toutes à même d'exploiter. Elles sont séparées des autres ensembles de communautés ou groupes de voisinage qui les entourent par une distance qui peut varier de deux heures à trois journées de marche. La formation de ces ensembles résulte généralement de la division, par fissions successives, d'une seule et même commu. nau té initiale.

A une échelle territoriale plus vaste, un certain nombre de communautés sont issues par fissions répétées d'une seule communauté initiale ou d'un mème groupe de voisinage constituant ce que $N$. A. Chagnon appelle un blocs de populations apparentées, et sont dispersées au sein d'un ensemble territorial distinct de celui du bloc voisin. Ce principe de formation des blocs de population n'exclut pas, à la frontière, les interférences avec les blocs voisins ${ }^{5}$. A l'intérieur d'un bloc, les relations entre communautés, comme celles qui sont entretenues avec les groupes voisins appartenant à un bloc distinct, peuvent ètre pacifiques ou hostiles.

Les relations d'alliance et d'amitié entre les groupes locaux s'établissent et se maintiennent grâce à des échanges de visites et de biens, à des invitations réciproques à des cérémonies funéraires et par le biais des échanges matrimoniaux. Elles se traduisent aussi par une certaine solidarité économique en cas de disette et/ou surplus et militaire dans les situations de belligérance. A la fois fonction de la distance généalogique et géographique et du type d'unité de réfé. rence, les relations entre corésidents, entre membres de communautés voisines ou entre membres de communautés distantes, présentent des degrés variables de solidarité et d'alliance et sont réglés par un certain rapport d'évitement et de crainte. A chacun de ces niveaux peut se rompre l'équilibre des rapports sociaux et intervenir la violence. Mais quel est le sens de cette violence sur laquelle est assise en grande partie intra-et inter-tribalement la réputation des groupes yanomarni?

La guerre est toujours la prolongation dhostilités déclenchées antérieurement ; elle est essentiellement une vengeance destinée à réparer une offense et à compenser un dommage affectif, plus particulièrement la perte ou l'atteinte grave de l'un d'entre eux par sorcellerie ou lors d'un combat.

L'existence et la formation d'un céthos, agressif et guerrier chez les Yanomami sinscrit donc dans le cadre d'un systeme vindicatoire. Une des principales composantes de l'éthique yanomami est celle de la rétorsion. L'esprit de vengeance est inculqué dès le plus jeune àge à l'enfant comme une valeur totalement positive et primordiale et la vengeance qui se réalise dans la plupart des cas sous la forme d'une destruction des choses et des ètres est légitime et légitimée. Elle exprime leur sens de la justice et elle obéit aussi à des motifs religieux : la vengeance 
représente un échange entre les vivants et les morts et ne peut être dissociée du culte des morts. Une fois entamees les hostilités, le couple agresseur-agressé est ambivalent dans la logique de la vengeance : qui se venge, car s'estimant agresó, devient agresseur pour l'autre et ainsi de suite. Comme, de plus, la compensation obtenue par la vengeance ne permet jamais de réparer totalement l'offense, on comprend qu il existe un climat permanent de persecution et d'agression qui se traduit sociologiquement par la suspicion, la rancune et l'hostilité mutuelles des parties qui ont ète une fois opposées.

Ce sont les hommes qui sont éduqués à combattre, à manipuler les armes et à mépriser la peur, le vrai courage consistant tout d'abord à donner des coups, mais encore à ne pas avoir peur d'en recevoir en retour. Tel est le véritable sens du terme waitheri, qui désigne les gens audacieux, combatifs, agressifs ou violents. II s'oppose aux conduites de peur, d'évitement, et de crainte (kirii) associées au genre féminin ${ }^{6}$.

Acéphale, cette société, faiblement hiérarchisée, est dépourve d'institutions d'autorité et répond par l'emploi de la force, la séparation et l'éloignement aux conflits qui surgissent. Autrement dit, la violence est un mode institutionnalisé de contröle social destiné à faire respecter le droit. Son usage modifie la composition et l'inscription territoriale des groupes locaux et l'orientation des réseaux d'alliance politique et matrimoniale. La violence est donc l'élément moteur de la dynamique des rapports sociaux inter- et intra-communautaires. Violence et guerre qui sont à l'origine des pratiques conjoncturelles et délimitées dans le temps révelent l'existence d'un rapport de force permanent. Ce rapport de force n'est pas seulement instauré par l'usage potentiel ou effectif de la force ouverte, mais aussi par l'usage de la force occulte. Violence manifeste et violence invisible ne peuvent, en effet, étre dissociées, et s'expriment au sein d'une seule et mème logique de l'agression. Considérée d'un point de vue structural, du plus proche au plus lointain, l'altérité sociopolitique chez les Yanomami se décompose ainsi en une série d'ensembles concentriques, chacun étant representatif d'un type d'échange de violence réelle et symbolique. Ces échanges contribuent à la for. mation de communautés installées dans un système dhostilitè chronique, latente ou manifeste, la hantise de l'agression, virtuelle ou réelle, orientant la grande majorité des comportements quotidiens.

Les Yanomami vivent très refermés sur leur propre communauté et développent parallèlement méfance et mépris envers tous ceux qui ne font pas partie de leur parentèle (cognats, divisés en consanguins et affins) ou de leur communaute d'appartenance (yafitheri ceux de l'habitation), y compris envers les communautés non-ennemies (nofi : amies), et cela d'autant plus si elles sont d'origine distincte. Cette dévalorisation d'autrui croit à mesure que l'on s'éloigne dans la parenté et dans l'espace. La terminologie de l'agression est d'ailleurs dérivée du terme nape $\hat{e}^{-7}$ désignant à la fois les étrangers et les ennemis. Il forme un couple d'opposition avec le terme yanomami qui désigne les êtres humains, et it recouvre une catégorie qui, sémantiquement, a valeur d'infra humanité.

Mais la violence peut s'exercer aussi à l'intérieur mème d'une communauté, entre parties préférentiellement alliées et aussi entre proches parents. $A$ tous les 
niveaux (individus et segment de parents, communauté, groupe de communautés), la position particulière au sein des réseaux d'alliance et de solidarité, fondée sur des relations de parenté et d'affinité mais ourrant la possibilité d'alliances pluri. directionnelles, fournit à chacun des moyens d'une part de protéger aes droits et de se venger des offenses et des dommages subis et d'autre part d'être aidé et défendu sil est lui-mème l'offenseur ou le transgresseur. En l'absence de liens de consanguinité ou d'affinité ou de relabions d'alliance et d'amitié longuement etablies, la violence semble s'exercer plus fortement, plus durablement et plus facilement aussi, entre groupes d'origine différente. En ce qui conceme les groupes de la Parima, les rares cas de massacre rapportés (liés à une traitrise lors d'une invitation ou à une mise à feu de (habitation) font intervenir des acteurs appartenant à des groupes d'origine distincte. Cependant si la guerre pratiquée en tre ces demiers peut varier de celle pratiquée à l'interieur d'un groupe d'origine commune, il s'agit d'une différence de degre, mais non de nature.

Les conflits entrainant l'usage de la violence sont principalement l'adultère, le vol de nourriture et de récoltes, de biens (et notamment le chien) et de femmes mariées, la destruction de plantations et de biens de toute nature et l'at teinte partielle ou totale à l'intégrité des chiens ou des personnes, par voie d'arme ou de sorcellerie.

Une série graduée de combats - duels, luttes rangées, raids - permettent de régier les comptes, autrement dit de faire payer le prix de la perte, de la douleur et du sang, et de tenter d'annuler l'effet de la colère (hushuo : ètre furieux/ètre affigé). Cependant, les vengeances n'empruntent pas seulement le moyen de la lutte ouverte; différentes pratiques de sorcellerie interfèrent dans le système vindicatoire. I semble que l'ensemble constitué par les combats ouverts caracteriserait davantage la tentative de réglement direct de conflits dans lesquels lídentité de l'offenseur est manifeste ou désignée, et que l'ensemble constitué par les agressions occultes renverrait pour sa part au règlement indirect de confits sourds, ou a la realisation de vengeances inextinguibles ou envers quelqu un dont on pense qu il est le responsable du dommage sans en être absolument certain. Dans les premiers, le reglement est réciproque, c'est-à-dire que la partie offensée admet le principe de la rétorsion, dans l'autre il est unilatéral, c'est-à-dire que la partie lésée cherche à éviter des représailles.

\section{LES COHBANS REGULIERS}

Les Yanomami pratiquent divers combats, en duel ou en bataille rangée, que lon peut classer en deux types distincts : les combats potikoi (frapper sur) et les combats au baton (noprushi) ou au casse-tete (fiimo). Il s'agit d'alfrontements obëissant à des régles précises auxquelles les parties concemées acceptent de se soumettre, sous le regand de la collectrité et dhommes influents veillant à la régularité des combats. 
Les combats patikai

Le combat potikai est un duel au cours duquel les coups sont alternativement donnés et reçus. Les combattants se frappent le mème nombre de fois jusqu'à ce que l'un d'entre eux se retire et soit immédiatement remplacé par un parent ou un allié.

Les coups sont portés sur le pectoral avec le poing, la paume ou à l'aide d'une pierre tenue dans la main ${ }^{8}$, ou sur le flanc avec la paume, plus rarement avec le plat d'une machette ou le dos d'une hache. Ces techniques de combat sont hiérarchisées en fonction de la douleur provoquée ${ }^{9}$, légère quand l'arme est le poing, pius forte et pouvant aller jusqu'à l'évanouissement dans les cas de duels à la pierre, à la machette ou à la hache. Il y a une corrélation entre les moyens utilisés, la classe d'àge des combattants et la gravité du délit ayant entrainé le combat. Les joutes sont exécutées par les hommes, les femmes participant par les encouragements ou les injures qu'elles prodiguent aux parties en présence. Les hommes murs indiquent aux combattants quand entrer dans le toumoi et quand en sortir en fonction de leurs meurtrissures (chacun doit recevoir autant de coups qu il en a donné à moins qu lil ne soit trop fortement blessé avant que tous les coups aient été rendus), comment se mettre dans la bonne posture pour recevoir le coup, et ce sont eux aussi qui font cesser les combats.

Ces joutes prennent souvent place au cours de fêtes (reafu) auxquelles communautés voisines et communautés lointaines, mais néanmoins alliées, se convient. Ces fêtes ont pour occasion une cérémonie funéraire au cours de laquelle une partie des cendres d'un défunt conservées dans des gourdes seront consommées, mélangées à une bouillie de banane, et au terme de laquelle les invités recevront un don de nourriture préparée. Le combat se déroule généralement après l'accomplissement du rite funéraire, parfois comme par jeu, en vue de se démontrer sa vaillance, mais plus fréquemment parce qu ì y a eu litige dans le passé entre hõtes et invités (calomnies, accusations, vol ou endommagement de récolte, d'un chien ou d'une femme, pratiques de sorcellerie, blessures ou homicides perpétrés lors d'une guerre passee...). Ce sont les hôtes qui frappent les premiers les visiteurs, plusieurs paires pouvant se battre simultanément, selon les cas. Les communautés qui se rendent à une invitation de ce type savent qu'un patikai peut lui ètre parfois associé, sans que cela implique une inimitié entre les deux groupes. La souffrance endurée et l'action elle-mème permettent de régler un différend tout en gardant ou sauvegardant des relations d'alliance et d'amitié, ce qu'exprime le contexte cérémoniel du combat. Pour le groupe offensé ne pas demander répara. tion, comme pour le groupe pris à parti ne pas accepter de combattre est faire preuve de pusillanimité et de couardise et par là-mème inciter à l'irrespect et l'agression à leur encontre, tandis qu'en se provoquant ils se prouvent mutuellement leur combativité.

Quand un groupe entend régler de la sorte un compte par un combat patikai, i) invite d'abord la communauté adverse ; puis cette dernière invite à son tour chez elle, ses hötes à participer à une fête au cours de laquelle a lieu un autre combat patikai pour qu ùls soient quittes et le litige réglé. Entre hôtes et invités, 
des luttes patikai sont possibles à tout moment, ̀̀ la suite d'un différend (accusations, mensonges, nom personnel prononcé, conduite provocatrice, problèmes avec des (emmes mariées...). Au moment du départ des invités, quand sont effectués des échanges de biens, il se peut qu une insistance trop grande de la part des invités à réclamer certains biens provoque une lutte patikai.

En dehors des fétes reafu, ces combats peuvent aussi avoir lieu entre communautés très proches ou mème entre corésidents afin de régler un conflit immédjat (pour vol, lorsqu'un homme en service de gendre dans la communauté de ses beaux-parents est rejeté par son épouse avec l'assentiment de sa belle-famille et qu'ul proteste quand elle a des rapports sexuels avec d'autres hommes du groupe, quand un homme a pris l'épouse d'un autre...). Lors de visites effectuées entre des communautés aux relations plus ténues, un duel patikai, suivi éventuellement de plusieurs combats se déroulant alors au sein de fêtes cérémonielles, peut avoir lieu entre deux parties hostiles dont l'une accepte de se rendre chez l'autre ou dans une communauté très proche. Le fait d'accepter de pratiquer ensernble ce type de lutte est la marque qu'elles ne souhaitent plus être ennemies et qu'elles ne se flècheront plus. Cela permet de rétablir, après d'anciennes hostilités, des relations jusque là gelées et d'entamer à nouveau des relations pacifiques.

\section{Les combats au bâton}

Une autre classe de combats e pratique à l'aide de bàtons noprushi, simples bois écorcés, longs ou courts, ou encore à l'aide d'un casse-tète plus élaboré en bois de palmier (fimo) doté d'un tranchant et pouvant faire office d'épieu ${ }^{10}$.

Un combat simple en duel se pratique essentiellement entre corésidents ou entre communautés très proches. Ces duels surviennent sur le champ lorsqu'on décourre un vol de nourriture ou un vol dans son jardin, lors d'une altercation si un nom personnel est prononcé, pour un adultère, pour un chien blessé ou tué, pour défendre une scur trop battue par son mari, ou lorsqu'un homme veut prendre pour lui une femme mariee ou veuve. Ce sont les hommes qui combattent ainsi mais, dans le cadre d'une querelle domestique, deux femmes peuvent aussi régler de cette façon leurs différends, en cas de jalousie ou de vol par exemple. Armé d'un bãton ou d'un casse-tète, l'offensé se dirige sur la place centrale de Ihabitation et invective l'offenseur. Si celui-ci relève le dét, il se présente à son tour avec un bäton sur la place. Is sont absolument immédiatement entourés et soutenus par des parents et des alliés qui accourent armés de haches, de machettes, d'arcs ou de bätons. A ce stade, la querelle peut se désamorcer sous le simple effet de la menace d'affrontement et les deux camps se disperser. S'lls décident de se battre, l'offenseur se met en position pour ètre frappé ; il incline la tēte et présente son cräne à l'offensé ${ }^{11}$. Blessé, il rend le coup sil en est capable, sinon un de ses proches se substitue à lui. En général, le combat cesse après que chacun ait été assommé. Les femmes empèchent souvent aussi en retenant le bâton qu'un deuxième coup soit donné. Le conflit est en principe définitivement réglé.

Lorsque le bàton est utilise à l'encontre d'une autre communauté, les combats revêtent en général beaucoup plus d'ampleur. Les hommes accompagnés des 
femmes se dirigent vers l'autre maison en brandissant leurs bàtons, entrent sur la place et demandent que les hommes du groupe offenseur se présentent. Ils sont plusieurs alors de chaque partie à se frapper, un coup contre un coup généralement, en se relaysnt. Les combats cessent quand plusieurs personnes sont blessées et ont perdu connaisance. Une tension extrême accompagne les duels au bâton ou casse-tête. Ces duels anglants peuvent très vite dégénérer gi l'un des combattants est gravement ou irrégulièrement frappé. Même si le choix de ce type de réglement répond au souci de contenir la violence, l'escalade peut à tout moment produire, créant entre les deux groupes des relations dhostilité plus graves encore. Les duels au bàton peuvent se terminer par une bataille rangée généralisée, les deux camps se frappant mutuellement de manière anarchique. S'il y a un mort au cours d'un combat, il s'ensurva un cycle de vengeance et la guerre. Dans un conflit intracommunautaire, cela implique qu'un des deux groupes part se réfugier en forêt ou dans une autre communauté.

Le cas de figure du combat en duel n'est pas toujours réalisé d'emblée. Au sein d'une mème maison, ou entre deux maisons distinctes, deux parties armées de bàtons peuvent s'affronter (yarikayou), un camp tapant et faisant reculer l'autre, puis inversement.

Ces combats prennent l'allure de véritables expéditions quand les Yanomami, armés de bâtons longs, vont affronter une communauté les ayant dépossédés d'une femme, nous détaillerons plus loin ce point, ou ayant détruit leurs planta. tions. Ce dernier motíf est considéré comme une véritable provocation. I est très fréquemment lié au premier : pour se venger, le groupe dépossédé va détruire la plantation du séducteur. Ce faisant, il arrive souvent quils se trompent de parcelle car ils ne connaissent pas bien les jardins des autres communautés; le groupe agressé va aller à son tour détruire d'autres plantations et plusieurs groupes se trouvent alors en position dhostilité ouverte. Le terme employé ici est similaire à celui des raids de guerre : napëhuu, aller en ennemi, ou wayuhuu, aller en agresseur, en guerrier. Chacune des deux communautés opposées demande renfort à d'autres communautés qui se joignent à elles le jour de la bataille et combattent à leurs côtés. Le groupe des offensés, femmes comprises, se dirigent vers la maison des offenseurs tôt le matin, ou bien souvent dorment en forêt près de la maison concernée et sortent au petit jour. Is sont peints comme pour un raid de guerre en couleur sombre (noir ou violet), armés de batons décorés et peints. Le groupe des offenseurs peut sortir de la maison à leur rencontse pour leur faire face (certains groupes vivant dans la Sierra Parima font ces combats en pleine savane, alleurs, un endroit dégagé est nécessaire) et s'aligne pour former l'autre camp. Les coups seront portés nimporte où, sur le cräne, sur le visage, les épaules ou les bras. Le camp des offensés fait reculer le camp adverse, puis les offenseurs répliquent et font reculer à leur tour les attaquants et ainsi de suite, jusquà ce quil y ait trop de blesses pour continuer. Quand le groupe local repousse ses adversaires avec suffisamment de succès pour leur faire abandonner la partie, la bataille cesse sous les diatribes des femmes des deux camps qui se défient et se menacent, les unes promettant qu ils reviendront plus nombreux avec de nou. veaux alliès, les autres quills seront prèts et n'auront pas peur de se battre. 
Selon le rapport de force en présence et le degré de colère de la partic lésée, il peut y avoir plusieurs batailles consécutives avant qu il soit procédé au réglement du litige par un combat en duel au bâton court. Exécuté à l'intérieur de la maison, ce dernier clôturera le différend si l'offenseur, ou l'un de ses représentants, accepte d'incliner le premier la tête, prêt à recevoir le coup de massue. $\mathrm{B}$ arrive que certains groupes défaits abandonnent la poursuite des hostilités après le premier combat. D'autres, en ee présentant pour combattre un groupe qui les a offensés transigent (car ces batailles sont très dangereuses et blessent grièvement) par l'intervention dhommes influents, médiateurs entre les deux parties. Is peuvent décider alors de reporter le combat lors de fêtes reafu : ils inviteront trois fois le groupe offenseur chez eux et combattront deux fois au poing, puis une fois à la pierre pour terminer. La règle exprimée par nos informateurs est d'effec. tuer d'abord deux batailles au bàton long avant de combattre une fois au bâton court. Mais certains vont se battre plusieurs fois ; d'autres encore, persuadés que le rapport de force leur est défavorable, rebroussent chemin avant d'arriver sur les lieux.

Quelle que soit leur issue, ces batailles peuvent aussi entrainer l'escalade des hostilités, un groupe rendu furieux en raison des blessures reçues pratiquant un raid contre l'au tre camp et déclenchant ainsi en retour des opérations belliqueuses.

Il est difficile de formaliser plus précisément le mode de compensation des dommages, différents facteurs intervenant simultanément dans ce système de répasation par les coups. L'offensé évalue lui-même la valeur de l'offense et le montant de la réparation. Ses propres sentiments jouent mais aussi les forces quil est susceptible de rassembler comme l'influence qu'exerce son entourage. A la fois, la distance généalogique et géographique entre les parties, l'état général des relations entre les personnes et les communautés, les événements extérieurs peuvent influer sur le mode et le degré des représailles exercées.

En général, pour un adultère, un ou plusieurs vols, un chien blessé ou tué, l'usage de charmes entre corésidents ou membres de communautés très proches geographiquement, on se bat en duel une fois au naprushi. Nous verrons plus loin ce qu il en est de l'enlèvement de femmes mariées.

Durant les trente mois (de mars 1980 à aoùt 1982) que nous avons passés chez les Yanomami de la Sierra Parima, il y a eu quatre cas ayant entrainé des batailles intercommunautaires au băton, tous à propos d'une femme (suwe nowë ha), dont un cas doublé de la destruction de plantations de bananiers et de taros (kuratha, ofina nowe ha). Les relations entre parties opposées reprennent plus tard quand les hommes influents décident de visiter les hommes de l'autre com. munauté et vont échanger des discours himou pour se réconcilier.

Cependant les hostilités ainsi développées, tout en permettant de ne pas passer à un mode plus radical de violence comme le fléchage et de conserver des relations relativement paisibles, marquent et cristallisent les conflits entre individus, entre factions et entre communautés. Les combats au naprushi, surtout quand ils sont le résultat de conflits intracommunautaires, président à l'éclatement des maisons communes et au morcellement des groupes locaux, et induisent le mécanisme d'atomisation qui provoque la multiplication de communautés indépendantes. 
En général, à la suite dhostilités ayant provoqué un ou plusieurs combats entre coresidents, une faction constituée par tous ceux qui se sont ralliés à la partie lésee fait la démonstration de ses sentiments en refusant de continuer à vivre dans le meme cercle dhabitation. Iss sinstallent un peu plus loin afin de se démarquer territorialement : si la distance n'excède pas dix minutes à une demi-heure de marche. ils seront toujours considérés comme faisant partie du mème ensemble de communautés. Shis vont s'installer à plus de deux heures et jusqu'à une demi-joumee de marche, cela indique une scission importante, une véritabie rupture des relations et ils ne seront plus considérés comme faisant partie du même ensemble de communautés. Ds peuvent aussi, s'ils sont trop peu nombreux pour rester isolés, rejoindre (worokei) des parents ou des alliés vivant dans une autre communauté à laquelle ils se joindront ou près de laquelle ils sinstalleront. Quand une scission se produit après des combats patikai ou au naprushi, deux factions corésidentes peuvent se séparer tout en restant voisines, tandis que deux communautés voisines se séparent plus nettement, ne faisant pius ainsi partie du même ensemble de communautés. Dès lors qu'elles sont séparées sur une base conflictuelle, les communautés craignent les pratiques de sorcellerie. Si la maladie ou la mort surviennent, elles peuvent se suspecter, ce qui entraine en général un cycle de représailles.

\section{Les femmes-njeu}

Les disputes au sujet des femmes sont avec les conflits liés au vol des récoltes les causes les plus fréquentes des combats au bàton chez les Yanomami. Ce sont elles qui entrainent le plus grand nombre de fissions au sein des communautés ou de séparations entre communautés proches. Nous ne pensons pas que le problème puisse ètre posé en termes de compétition pour les femmes ou que la fréquence des conflits tienne à l'insuffisance du nombre de femmes disponibles ou encore que les raids soient motivés par le rapt des femmes.

Contrairement à une idée répandue, les Yanomami ne font généralement pas la guerre pour obtenir des femmes. Dans les groupes de la Sierra Parima, le rapt de femmes ne se pratique pas entre ennemis. Leurs voisins méridionaux immédiats, ceux qu'ils dénomment Shamatari, ont enlevé quelques femmes durant une longue guerre qui les a opposés dans le passé, mais pour ces derniers non plus le rapt de femme n'est pas le but premier des raids qu' 'ils intentent. Il est tout au plus, avec les quelques biens qui peuvent parfois ètre annexés, un bénéfice secondaire. Il est surtout une des formes de la pratique vindicatoire. De plus, comme les femmes raptées tentent généralement de s'enfuir, soit immédiatement durant le trajet de retour du raid, soit plus tard une fois installées et mariées, un nombre minime d'entre elles vient effectivement accroitre la capacité de reproduction du groupe ravisseur.

Par ailleurs, l'emploi du terme de compétition, sémantiquement associé à une notion de concurrence et de rareté, biaise la compréhension du phénomène guerrier dans la société yanomami. En effet, les Yanomami se battent lorsqu'on les prive des droits qu ils détiennent sur une femme, comme lorsqu'on les prive des 
droits qu'lis exercent sur des bananiers ou sur un chien, et ils affirment aussi quils se battraient si un groupe les provoquait en venant chasser ou 8 installer sans leur accord sur le territoire qu ìs exploitent. Mais il ne s'agit pas là à pro. prement parler d'une compétition pour obtenir des fermes, des biens ou des terres fertiles soumise à la loi de la rareté. La violence répond dans tous les cas à une offense et à la privation d'un droit d'usage potentiel ou effectif.

I faut distinguer l'objet en cause dans la dispute, ou l'objet de la transgression, de la nature de la transgression elle-mème qui est, elle, la véritable cause des formes de violence exercées dans la société. Dans le cadre des disputes intervenant à propos des femmes, le motif qui provoque les combats correspond à la violation d'un droit matrimonial en exercice.

I] faut considérer plusieurs cas de figures de ce que les Yanomami nomment suwè nowe ha (à cause d'une femme) et qui place sous une même catégorie tous les conflits liés à la question de l'appropriation des fernmes par les hommes, question, en fait, qui est aussi celle de la rupture par les femmes du lien matrimonial.

Premier cas, une femme, mariée ou veuve, s'enfuit en forèt avec un homme appartenant à sa communauté ou à une communauté voisine pour imposer le fait de leur liaison à son épouse ou à la famille de son mari défunt. L'affaire se règle à leur retour : si l'époux est décédé ou si elle vit séparée de son mari depuis longtemps, la partie offensée peut demander que le combat s'effectue au poing. Mais si le mari exige le retour de sa femme au foyer et qu'elle refuse de lui revenir pour rester avec son amant, le duel entre le mari et l'amant se fera au bãton. Notons qu'à son retour, la fermme est généralement frappée par son mari, brülée avec du bois du foyer ou piquée avec une pointe de fleche ou une pique, mème dans les cas où elle veut bien revenir avec lui, ce qui consomme souvent la rupture.

Deuxième cas, un homme prend une femme mariée dans la communau té de ses parents ${ }^{12}$.

Troisième cas, plus complexe, une femme marièe fuit (shuuafiayou, quitter, fuir sa résidence) la communauté de son mari pour rejoindre généralement une communauté où elle compte de la parenté. Là, elle ne tarde pas à être prise, parfois après avoir été violée par tous les hommes qui ne sont pas ses consanguins classificatoires, par un homme de cette communauté ou d'une communauté voisine. En quittant sa residence, elle agit de son propre chef soit qu'elle veuille simplement rejeter un époux avec lequel elle ne s'entend pas et qui l'a battue, soit que, conquise, elle désire rejoindre un homme précis. I se peut encore qu'elle soit séduite et s'enfuit pour suivre un visiteur ou bien un homme d'un groupe invité à vivre quelque temps avec sa communautè.

Ce sont ces derniers cas qui provoquent principalement les grandes batailles au naprushi si la fermme n'est pas rendue au mari. La partie offensée, armée de batons, vient la rechercher. On essaye alors de la tirer hors de la maison tandis que les femmes de cette maison la retiennent par le bras (shoiai). Elle est ainsi traillée, écartelée, comme dans la lutte à la liane (tug-of-war), entre deux camps qui luttent, l'un pour la récuperer, l'autre pour la gander. Lintervention d hommes influents peut éviter les heurts. Mais si l'homme qui s'est empare de la femme 
refuse de la restituer, ses parents et alliés repoussent les assaillants à coups de bātons hors de lhabitation. Quand la femme est reprise ou bien rendue lors de l'assaut, elle est très souvent fortement chàtié, et il est des cas où elle succombe aux coups. Si la première tentative pour reprendre la femme échoue, une autre bataille rangée a lieu une fois guéries les blessures du premier affrontement.

Ils peuvent ainsi s'affronter plusieurs fois jusqu'à ce que les offensés s'estiment venges. Des intercesseurs, des hommes qui peuvent être partie prenante pour les offensés mais en relation d'alliance avec le groupe infracteur, peuvent proposer un combat en duel à l'intérieur de la maison afin que la colère de l'offensé perde sa force, se dissipe, et que des rapports d'amitié puissent se rétablir. Lors de ce règlement de comptes, la fernme qui a tait l'objet de la dispute, si elle ne s'enfuit pas, est maintenue de force debout, par les hommes, les bras en croix pendant qu'on lui assène plusieurs coups de bàton (trois en général) sur le cràne. Quant à l'amant, et à son groupe, ils acceptent d'ètre les premiers à incliner la tête pour recevoir les coups. Cette effusion de sang permet alors de calmer la colère du mari dépossédé et de la rendre okewẽ, sans effet.

Les disputes à propos des femmes sont donc bien chez les Yanomami les premières causes de scissions importantes intra- et intercommunautaires et elles sont bien aussi les motifs les plus tréquents qui peuvent provoquer, sil y a escalade de la violence et qu'un fléchage ou une mort interviennent au cours du reglement de comptes, des hostilités belliqueuses entre les parties. Mais nous ne pouvons pas dire pour autant que ces motifs sont uniquement et directement ceux qui déclenchent le plus fréquemment les guerres, car un des tout premiers motifs des guerres est probablement l'atteinte d'un individu par sorcellerie, l'autre face du système d'agression dans la société yanomami dont nous allons maintenant traiter.

\section{LES AGRESSIONS OCCULTES}

Le mal, le mal-être, la maladie et la mort sont très fréquemment imputés à l'action malveillante d'autruj qui prend la forme d'une agression de type magique. La crainte de la sorcellerie est profondément ancrée dans la vie quotidienne des Yanomami car sa pratique est supposee courante. Elle est pour eux une des principales formes de criminalité.

Il n'existe pas de sorcier en tant que personnage particularise chez les Yanomami : tout le monde peut s'adonner à un moment ou à un autre à la sorcellerie. Ainsi, quoique de manière plus limitée, les femmes ont aussi accès aux gestes qui portent atteinte à l'intégrité physique des individus. Chacun des charmes (fëri) employés provoque des symptōmes particuliers et s'emploie pour un motif precis : jalousie, vengeance sexuelle, séduction, haine... Is sont utilisés selon trois techniques différenciées qui correspondent aussi à la distance spatiale entre les personnes concernées. La mise en contact peut se faire tout d'abord directement sur la personne par projection, frottement, saupoudrage des fêri ou bien encore en les mélangeant à de la peinture corporelle ou à de la nourriture. Elle peut se faire aussi en frottant entre les paumes de la main, mélangées à des charmes, la terre contenant la trace ou l'empreinte que le pas a laissée dans le sol, 
une chique de tabac, ou des détritus sucés par l"individu ou encore en frottant la trace que l'on a fait mordre par un serpent. Celte pratique est dite raraprai. On peut enfin souffler (forai) à l'aide d'une sarbacanne (yoroama) des charmes sur une personne, un jardin ou une habitation.

La première technique est employée à l'encontre de personnes que l'on côtoie et que l'on peut donc atteindre aisément : des corésidents, des voisins proches ou des gens de passage. Elle vise à causer dommage, à rendre malade, mais pas vraiment à supprimer la personne. Elle est la plus facilement et la plus communément pratiquee. Chacun possède dans son jardin plusieurs plantes magiques qui servent à fabriquer des charmes bénéfiques (favorisant la chasse de certains gibiers, la croissance, l'ardeur au travail. la séduction ou son évitement...) ou maléfiques (contre les incurseurs, les gens détestés, les femmes qui se sont refusées...). D'autres substances, d'origine végétale ou animale, sont prélevées dans la nature. Les femmes cultivent aussi quelques plantes fëri qu'elles emploient au gré de leurs haines, de leurs jalousies ou de leurs désirs. certaines particulièrement contre les hommes. Contre les femmes, ces derniers fabriquent aussi des chames pour provoquer la consomption et la sterilité. Des charmes mortels peuvent ètre employés à l'encontre de visiteurs, mèlangés à la soupe de banane qu 'on leur offre. Lors des combats, comme nous le verrons plus loin, ils peuvent ètre tenus dans le poing ou frottés sur les armes comme la pierre, le bàton ou le casse-tète. Ils sont encore fixes sur les pointes de fleches utilisees contre les ennemis.

La deuxième technique (roraprai) est plus difficile à pratiquer, elle nécessite d'avoir à sa disposition un support intermédiaire, substitut de la personne. Elle s'accomplit à distance, nuitamment et secrètement, chez soi, et elle vise à détruire physiquement la personne. Une fois decouvert, un tel geste peut entrainer des teprésailles et un cycle de vengeance. Il est essentiellement masculin et peut s'exercer à l'encontre des hommes comme des femmes. L'empreinte ou la trace est prélevée dans le sol après le passage de la personne, dans son jardin particulierement. Lorsqu'on habite trop loin, on peut demander à un complice de se procurer la terre contenant l'empreinte de la victime de son choix. On cherche ensuite en forét un serpent auquel on fait mordre le morceau de terre qui a été mélangé à des fëri puis pétri. Un autre serpent mordra à distance la victime. La terre est encore frottée deux nuits de suite pour garantir l'effet mortel. Sans la morsure du serpent, la terre doit ètre malaxée et effritée une dizaine de nuits pour être efficace. Elle est partagé en deux morceaux et plusieurs personnes dans deux villages distincts peuvent participer la nuit à l'ensorcellement en mélangeant à la terre des fêri. Chaque jour la victime maigrit un peu plus et au bout d'une dizaine de nuits succombe.

De la mème manière, en temps de guerre, une chique de tabac oublièe sur les lieux de campement ou d'embuscade par les guerriers peut être récupérée afin d'être coupée en deux et frottée avec des feri mortels. Deux villages peuvent ainsi se livrer à un ensorcellement qui sera fatal au guemier étourdi.

Cette technique d'agression se pratique entre ennemis mortels, appartenant à des ensembles de communautés différents. Elle n'est pas utilisée à l'intérieur dune maison commune ou entre très proches voisins (ils savent que cela 
entrainerait le đéchage). Dans ce casłlà, ił existe une formule plus douce (si irurukel) : à la motte de terre mélangée à des fëri sera intégré un os de singe, ce qui provoquera une douleur aiguè à la cuisse de la victime par exemple, mais ne pourra la tuer.

Les pratiques raraprai sont bien souvent à l'origine des discordes intervenant entre groupes voisins lorsqu'un groupe suspecte un autre d'ètre la cause de la mort d'un de ses membres. Ces soupçons peuvent se concrétiser longtemps après le décès, quand quelqu'un rapporte le nom du meurtrier. De ces accusations de sorcellerie raraprai, on demande raison lors de discours formels, et de leur fait l'on se bat au poing ou à la pierre au cours des fêtes cérémonielles, le groupe atteint invitant le groupe soupçonné.

Quand l'accusation porte plus précisément sur quelqu'un, ce dernier peut ètre invité par le messager venu au devant des invités à se rendre dans la maison pour chercher de la nourriture, puis, sur place, pris à partie par les parents du mort et détie dans un combat sanglant. Dans un tel combat, les deux hommes se placent face à face, l'un contre l'autre, et s'enfoncent dans le dos une arme confectionnée à l'aide de trois pointes d'os taillé emmanchees dans un bois ou le tranchant d'un éclat de bois de paimier, d'une hache ou d'une machette. Un combat patikai est effectue ensuite avec les autres membres de son groupe.

Quand les Yanomami pratiquent cette lutte au corps à corps pour se blesser (akriai), ils font montre de leur intense colère en reproduisant le combat mythique de Coati et Jaguar, deux êtres des plus agressifs et également combatifs; combat symbolisant de ce fait une charge suprème de violence.

Dans de nombreux cas encore, les soupçons portent sur un groupe avec lequel les relations sont déjà distantes à cause de conflits passés. Lorsqu un ou plusieurs morts leur sont attribués, la vengeance s'effectuera alors de manière occulte selon le mode raraprai ou selon le mode forai. Si ces techniques de destruction s'avèrent inefficaces aprés plusieurs tentatives, on procédera à des représailles à visage découvert avec des arcs et des flèches. Il se peut aussi qu un groupe, convaincu de l'identité du ou des meurtriers, se livre directement à un raid.

La technique forai s'assimile davantage que les autres pratiques de sorcellerie à une technique d'agression guerrière. Elle est rarement pratiquée individuellement. De véritables expéditions sont organisées (okrahuu : aller en sorcier, okra) par quelques hommes, trois ou quatre, partant de nuit sans prévenir leur communauté. Seuls quelques hommes importants sont mis dans le secret. Leurs agresseurs se peignent en noir, comme pour les raids armés, mais partent désarmés. Ils n'emportent avec eux qu'une ou deux sarbacanes et un paquet de feuilles contenant les fêri. Quand ils atteignent la résidence de leur victime, située à un, deux, trois jours de marche, ils s'embusquent dans des broussailles pres des chemins de passage, ceux qui débouchent sur le jardin particulièrement, car de cet endroit ils pourront distinguer leur victime. Is vont souffler à tour de tòle et de loin les charmes que chacun a préparés. Ceux-ci atteignent leur but, qu 'ils passent devant, derrière ou au-dessus de la téte de la victime, ou qu'ils soient projetés la nuit sur le jardin, la forèt ou thabitation, faute d'avoir vu quelqu'un dans la journée. Quand ils effectuent plusieurs raids sans apercevoir de cible, ou lorsque les feri 
demeurent sans effets, ils transforment slors leur action en hostilités ouvertes, armés de leurs arcs et de leurs Dèches. L'échec des pratiques de sorcellerie et la nécessité de leur répétition est justifiée. D'une part, le pouvoir délétère des feri peut être annihilé par l'intervention des chamanes capables lors de la cure de déglutir les fëri, armes du crime. D'autre part, le fëri employé cette fois-là peut manquer d'efficacité - preuve en est qu'en d'autres expériences il est efficace - et laisser à la personne une chance d'en réchapper.

Une autre forme de chamanisme est mise, celle-là, au service de l'agression. Elle n'est pratiquée que par quelques-uns des chamanes, les plus puissants, ceux qui sont capables de déchainer leurs foudres sur les plantations et sur les habitations, de déclencher des accidents en forêt, et de provoquer les bourrasques de vent et les tempètes qui déferlent brusquement. Is dérobent, lorsqu ils se déplacent sous forme d'esprit, les ârnes des enfants pour les rendre malades et les faire mourir. Les chamanes opèrent aussi lors des rites d'agression et des raids pour garantir le succès d'une expédition et faire échouer celles de leurs ennemis.

Le chamanisme en tant que moyen de destrurtion se pratique généralement contre des ennemis virtuels, communautés lointaines dont on connait l'existence sans avoir de relation effective avec elles, ou contre des communautés avec les. quelles l' inimitié perdure, des communautés par exemple qui ont dû par le passé se déplacer à plusieurs journées de marche, à la suite d hostilités. En ce sens, le chamanisme d'agression est une relation d'échange symbolique nouée avec l'Autre, le plus lointain des Autres précisément connus, et signifie la reconnaissance sociale de cet Autre.

Audelà de cette sphère dinteraction, les Yanomami méconnaissent relativement les gens et la région qui les entourent. L'agression et l'échange de violence symbolique se prolongent par le biais de l'agression du double animal, analogon vital de chaque Yanomami dont Thomicide équivaut a provoquer la mort de l'ètre humain qui lui correspond. Les doubles d'un groupe sont supposés vivre dans une de ces régions habitées par des Yanomami inconnus tandis que les doubles d'autres Yanomami peuvent demeurer - pas nécessairement de manière symétrique - dans leur propre forêt.

Les différentes techniques de destruction occulte ne sont pas exclusives l'une de l'autre et peuvent se recouvrir partiellement. Leur emploi est fonction du degré d'éloignement et dhostilité des parties en présence. Dans le cadre d'une typologie schématique, on pourrait dire que la sorcellerie ordinaire est celle que l'on pratique surtout ich la sorcellerie de type roropai celle que l'on pratique là et parfois là-bas. que la sorcellerie de type forai est d'usage parfois lò mais surtout là-bas tandis que le chamanisme d'agression se pratique là-bas et surtout ailleurs. L'attaque d'un double aninal est, quant à elle, généralement fortuite et concerne exclusivement l'ailleurs.

Comme nous l'avons montré [C. Alès et J. Chiappino 1981-1982 et 1983], la maladie et la mort sont vécues comme une agression venant de l'extérieur et participent du système qui génère la représentation de l'agression chronique. La douleur, le malêtre et la maladie, événements dysharmoniques pouvant entrainer la mort correspondent dans le système des représentations à une conception 
demeurent sans effets, ils transforment alors leur action en hostilités ouvertes, armés de leurs arcs et de leurs Alèches. L'échec des pratiques de sorcellerie et la nécessité de leur répétition est justifiée. D'une part, le pouvoir délétère des fêri peut ètre annihilé par l'intervention des chamanes capables lors de la cure de déglutir les ferr, armes du crime. D'autre part, le fëri employé cette fois-là peut manquer d'efficacité - preuve en est qu'en d'autres expériences il est efficace - et laisser à la personne une chance d'en réchapper.

Une autre forme de chamanisme est mise, celle-là, au service de l'agression. Elle n'est pratiquée que par quelques-uns des chamanes, les plus puissants, ceux qui sont capables de déchainer leurs foudres sur les plantations et sur les habitations, de déclencher des accidents en forêt, et de provoquer les bourrasques de vent et les tempètes qui déferlent brusquement. Ils dérobent, lorsqu'ils se déplacent sous forme d'esprit, les ames des enfants pour les rendre malades et les faire mourir. Les chamanes opèrent aussi lors des rites d'agression et des rajds pour garantir le succès d'une expédition et faire échouer celles de leurs ennemis.

Le chamanisme en tant que moyen de destruction se pratique généralement contre des ennemis virtuels, communautés lointaines dont on connait l'existence sans avoir de relation effective avec elles, ou contre des communautés avec lesquelles l' inimitié perdure, des communautés par exemple qui ont dú par le passé se déplacer à plusieurs joumées de marche, à la suite d'hostilités. En ce sens, le chamanisme d'agression est une relation d'échange symbolique nouée avec l'Autre, le plus lointain des Autres précisément connus, et signifie la reconnaissance sociale de cet Autre.

Au-delà de cette sphère dinteraction, les Yanomami méconnaissent relativement les gens et la région qui les entourent. L'agression et l'échange de violence symbolique se prolongent par le biais de l'agression du double animal, onalogon vital de chaque Yanomami dont thomicide équivaut à provoquer la mort de l'être humain qui lui correspond. Les doubles d'un groupe sont supposés vivre dans une de ces régions habitées par des Yanomami inconnus tandis que les doubles d'autres Yanomami peuvent demeurer - pas nécessairement de manière symétrique - dans leur propre forèt.

Les différentes techniques de destruction occulte ne sont pas exclusives l'une de l'autre et peuvent se recouvrir partiellement. Leur emploi est fonction du degre d'éloignement et dhostilité des parties en présence. Dans le cadre d'une typologie schématique, on pourrait dire que la sorcellerie ordinaire est celle quel'on pratique surtout ich la sorcellerie de type rorapai celle que l'on pratique là et parfois lábas. que la sorcellerie de type forai est d'usage parfois là mais surtout là-bas tandis que le chamanisme d'agression se pratique là-bas et surtout ailleurs. L'attaque d'un double animal est, quant à elle, généralement fortuite et concerne exclusivement l'ailleurs.

Comme nous l'avons montré [C. Alès et J. Chiappino 1981-1982 et 1983], la maladie et la mort sont vécues comme une agression venant de l'extérieur et participent du système qui génère la teprésentation de l'agression chronique. La douleur, le malêtre et la maladic, événements dysharmoniques pouvant entrainer la mort correspondent dans le systeme des representations à une conception 
persécutive du mal. Les objets et les facteurs pathogènes renvoient toujours à une puissance hostile en action (chamane ennemi, esprit malfaisant, démon ou sorcier) qui est le véritable agent du mal.

Dans la vie quotidienne, les Yanomami gardent en permanence à l'esprit le fait qu ils peuvent ètre frappés sans qu ïls en soient conscients et tiennent comple de cette éventualité lors de tout déplacement proche ou lointain. Dans leur habitation comme en forèt, ils sont attentifs à tout bruit pouvant suggérer la présence de sorciers ôka ou de guerriers wayu. En visite, ils se méfient des aliments offerts et craignent qu'on prenne leur empreinte dans la terre (mayz yurarei). Dans cette logique de pensée fondée sur le principe de rétorsion et de non-compensation définitive de la vengeance, on comprend que tous les conflits intra- et inter-communautaires, quils aient été officiellement réglés par une dispute, un combat ou une fission, et toutes les agressions passées, entrainent un sentiment de persécution. Cette crainte des pratiques occultes d'agression déterminent et singularisent les conduites d'évitement ou à l'inverse de cótoiement de chaque individu à l'égard de chacune des autres communautés.

La sorcellerie, autant que la menace d'attaque armée, organise donc l'inscription dans l'espace, l'évitement et la mise à distance entre individus et entre groupes.

\section{VIOLENCE ET DISTANCE SOCIALE}

Outre les cas que nous venons de voir où la mort est attribuée à un acte de sor-

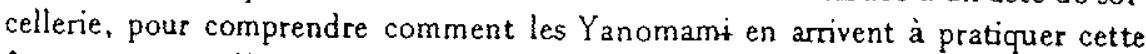
forme extréme d'agression qu'est le raid, il faut prendre en considération le fait que la violence peut échapper au contröle auquel on tente de la soumet tre dans le cadre des combats réguliers, I'hostilité sintensifier et entrainer l'utilisation de l'arc et des flèches.

Au cours d'un combat, si un coup irrégulier est donsé ou un participant griève. ment blessé, l'un de ses parents peut utiliser un épieu ou une hache pour le venger sur le champ. Il anive aussi que les duels au poing, à la pierre ou à la massue, provoquent la mort de l'adversaire ${ }^{33}$. Ainsi, quand nous avons commencé notre recherche sur le terrain en 1980 , les groupes de la Parima A venaient d'entrer en guerre avec les groupes de la Parima B. Lors d'une visite effectuée dans les com. munautés de la savane de niyayopé un groupe avait volé des fruits de palmiers dans une plantation rencontrée sur le chemin. Une querelle éclata et entraina tout d'abord un combat au poing, puis, la tension ayant monté, les hommes provoqués par le vol, fous de rage, relancèrent le combat avec des bâtons. Un des visiteurs succomba aux coups. C'était le signal de l'ouverture d hostilités guerrières qui, à mon départ en 1982. avaient déjà provoqué la mort de trois hommes de part et d'autre. Dans ce cas, comme pour chaque mort attribuée à autrui, la réparation sera obtenue en causant un dommage au moins équivalent au groupe des agresseurs. Au cours des cycles de vengeances, les blessures par fléchage - léthales ou non - sont soigneusement comptabilisées, tout comme les attaques de maison (shurukei) sur lesquelles, en désespoir de cause, faute d'avoir rencontré l'ennemi, les guerriers envoient une volée de flèches. 
Chacun des crimes influence la mise en cuvre des raids et contre-raids qui sont tentés de part et d'autre, de méme que l'identité des raideurs et des meurtriers ou celle de leur communauté d'appartenance influe sur le choix des hommes et des maisons ciblées.

II semble que provoquer le départ d'un groupe (yashuprai) correspond à sanctionner socialement le groupe chasse tandis que se séparer d'un groupe (sherereprai) prend le sens d'une sanction exercée à l'encontre des corésidents ou des voisins de la part de ceux-là mème qui s'excluent.

Fissions et séparations obéissent à un mécanisme complexe qui est aussi fonction de la taille de la communauté et de l'état de ses relations avec les groupes voisins. Une situation de belligérance intense incitera les Yanomami, au-delà des dissensions internes, à rester regroupés au sein d'une même habitation ou à $s$ installer à courte distance. Du fait de disputes incessantes, une faction peut aussi décider de se séparer du reste du groupe avant que les tensions internes ne deviennent trop fortes et ne provoquent des hostilités marquées. Et sils sinstallent à proximité, ils restent suffisamment liés pour se prêter main forte en cas d'attaque extérieure. D'autres se séparent encore car ils sont en désaccord a propos d'un conflit qui oppose leurs corésidents à une autre communauté. Is évitent ainsi de participer aux combats et d'être la cible des opposants lors des raids. La distance spatiale entre les maisons yanomami traduit le degré d'autonomie des actions et la souveraineté des décisions : plus on s'écarte, plus on rompt les solid arités, et inversement quand on se rapproche.

Chaque étape dans la séparation spatiale, aussi minime soit-elle, est fortement codée : plus la relocalisation est proche, plus cela signifie que la relation d'amitié est grande ; plus elle est éloignée, plus cela signifie que la relation d'hostilité est forte. La variation des distances entre les différentes unités résidentielles s'exerce à l'intérieur d'une gamme très fine : toute distance intermédiaire entre deux cercles dhabitation, qu'elle soit de quelques mètres ou qu'elle s'évalue en heures de marche, permet de mesurer les relations d'amitiẻ et caractérise, quelle que soit la nature des relations actuelles, le passé dinimitié et d'hostilités entre deux groupes. On voit donc que l'éloignement entre les différents groupes structure les relations sociales et ainsil l'un des buts de l'èchange de violence et de la guerre est de produire de la distance sociale entre soi et ses ennemis.

Dl faut insister sur le fait que les Yanomami ne se déplacent ni ne changent de territoire d'exploitation aisément. Hormis de rares cas de mouvements plus importants qu'à l'accoutumée pour sinstaller dans une région plus fertile. les Yanomami ne se déplacent que très progressivement pour implanter leur maison à proximité des nouveaux jardins et des territoires qu ils exploitent. C'est V. A. Chagnon [1968a] qui a le premier distingué ce qu'il a appelé les micro. moves et les macro-moves, respectivement les deplacements au gré des planta. tions horticoles et les déplacements effectués sous pression militaire allant d'une à trois joumées de marche. Seuls, en effet, des comportements agonistiques de la part de leurs corésidents ou de leurs voisins peuvent inciter les Yanomami à se réinstaller ailleurs, et seule la crainte des flèches, une guerre intense décimant plusieurs membres de leur groupe, peut les pousser à effectuer un déplacement 
correspondant à une journée de marche. Là, si les hostilités ne cessent pas et que le nombre de morts s'accroit, il sera nécessaire de mettre une distance beaucoup plus importante, correspondant à deux ou trois journées de marche, entre leur lieu de residence et celui de leurs ennemis mortels.

Au cours des guerres, les coalitions peuvent être relativement importantes, rassemblant les membres de six à huit communautés distinctes, et des troupes comprenant jusqu'à une cinquantaine de guerriers. Les communautés coalisées, lorsqu'elles sont proches, ont tendance, pendant la durée des hostilités, à se rapprocher sensiblement les unes des autres, pour assurer leur sécurité. Elles peuvent ainsi présenter rapidement un meilleur rapport de force en cas d'attaque et relayer pour effectuer des tours de guet. Elles se concertent outre pour toute activité guerrière, pour toutes les activités économiques qui les amènent à s'éloigner des maisons comme les expéditions de chasse qui durent plusieurs jours et surtout, les établissements périodiques en camp de foréts.

Les périodes de nomadisme sont déterminées par l'importance des ressources disponibles en forèt, mais, en période de raids intenses, elles permettent d'éviter les attaques et de décourager l'ennemi qui effectue des raids en vain. Les communautés de faible taille peuvent rejoindre une autre maison ou bien se regrouper avec d'autres dans une seule maison ou un seul camp de forèt. Après avoir fusionné, les communautés peuvent se séparer à nouveau et redevenir indépendantes une fois les hostilites estompées.

Mais la guerre chez les Yanomami, au-delà de conséquences centripètes ponc. tuelles gommant les dissensions internes passées et présentes, exerce principalement une action centrifuge sur l'organisation de la société, les groupes les plus faibles préférant finalement s'éloigner plutōt que de tenir tête.

\section{PAROLE, YIOLENCE ET CONTROLE SOCIAL}

\section{La médiation de la parole}

La parole, autant que la violence, structure l'ordre social chez les Yanomami. Dans le règlement des conflits, elle est utilisée comme instrument de médiation jusqu'au point où s'emploiera la force. I convient donc d'aborder brièvement ce sujet.

A l'intérieur de la communauté yanomami. il existe deux formes de prise de parole ayant pour objet de régler les différends ou de les prévenir : hirai, crier, vilipender ${ }^{14}$ et kanoamou, discours en forme de monologue pour informer, éduquer, influencer, se plaindre ou haranguer.

Cette dernière forme de prise de parole est le fait des pata (aines, grands hommess) et elle peut s'adresser aux membres de leur faction, ou plus largement, à l'ensemble des membres de la maison commune. Ce type de discours traite des affaires internes des groupes de résidence et aussi des affaires intercommunautaires. Il vise à influencer plus qu'à imposer : chacun reste libre d'adhérer ou non aux avis exprimés et de livrer des opinions differentes. Il permet $d$ harmoniser les décisions et de coordonner les actions communes, principalement 
pour les activités économiques et les mouvements collectifs. Le discours konoamou transmet des informations, des souhaits, des désirs, des frustrations ou des griefs, mais peut inclure aussi des mises en garde, des accusations, des dénégations. A travers lui, on se défend en cas de dispute, on répond à des calomnies, à des imputations de vol, d'affaires sexuelles, à l'intérieur de la communauté ou, dans le cadre de différendo inter-communautaires ${ }^{15}$.

La prise de parole hirai ou kanoamou constitue un puissant moyen de contröle social, car c'est le lieu où se jouent l'appel et le rappel à l'ordre. Sa fréquence et limportance de son rôle temporisateur varient toutefois en fonction de la taille des maisons communes et du nombre de factions en présence, la probabilité d'émergence de désaccords ou de conflits étant plus forte dans les maisons abritant plus de soixante personnes.

Entre communautes, les Yanomami pratiquent plusieurs types de discours formels lors des visites et des fètes qui leur permettent de régler des différends. comme de créer, maintenir et réaffirmer des relations pacifiques d'alliance et d'amitié. Himou est un discours diurne tenu alternativement et publiquement par deux hommes qui se tiennent près du foyer. Motivé par une raison précise. il a pour but dinformer, de discuter de projets militaires ou d'aviser des positions de chacun dans le cadre d'un différend qui oppose des parties que chacun représente (affaires sexuelles entre gendre et beau-père par exemple pour un cas de noshi imapou : refus de donner une fulle ou une sceur au mari). I permet d'exprimer le mécontentement et de pëyëprai - disputer, accuser, menacer - dans les cas de mensonges (horemou), de paroles rapportées (wanomou) qui ont suscité des problèmes, et de divulgation du nom personnel (sipoai).

Lors des fêtes, des duels oratoires nocturnes wayamou se déroulent jusqu'à l'aube avec une succession d'orateurs sur la place centrale. Is concement plus spécifiquement l'échange de biens, de nouvelles et d'amitiés. Mais au cours de ces discours, les Yanomami peuvent aussi pēyēprai lorsqu'il y a un contentieux. Cela peut se produire encore quand un homme, sur la demande de ses hotes. vient en émissaire chercher de la nourriture avant que le groupe entier ne se présente pour danser et ne soit accueilli. Dès son arrivée sur la place, cet homme entame un duel oratoire avec un ou plusieurs hótes au cours duquel ces demiers manifestent leurs griefs et pèyëprai.

Quand la fète se termine par des combats patikai, hòtes et visiteurs peuyent effectuer immédiatement après, par paires, un discours de réconciliation, de promesse d'alliance et d'échange de biens haohaomou ou encore yaimou. Tous ces discours ont une tonalité, un rythme et une gestuelle formelle et font appel à des références symboliques dont seuls les hommes mûrs ont la réelle maitrise. Les femmes sont totalement exclues de ces pratiques oratoires.

L'ensemble de ces discours publics, obéissant à des normes déterminées. contribue à la régulation des comportements de chaque individu à l'égard de ses corésidents ou à l'égard des voisins avec lesquels il entretient des relations d'alliance et d'amitié. Ils permettent de formuler plaintes et reproches, de s'attaquer verbalement, tout en évitant la détérioration des relations. Quel qu'en soit l'objet (demande de biens ou demande de comptes), ils correspondent à ce qu'on 
pourrait appeler un plaidoyer : chacun défend sa cause, dénie les accusations, accuse à son tour, contoume les problèmes et argumente sa propre version des faits en en manipulant habilement les paroles pour soutenir sa cause, son opinion ou une personne. Les discours sont à la base du maintien de relations pacifiques. C'est pourquoi les échanges de discours sont systématiques lors des risites que se font des hommes appartenant à des ensembles différents de communautés, et de nombreux échanges de discours président toujours à l'ouverture des relations d'anitié, comme à leur reprise, à l'isere dhostilites. On voit donc que dans cette société acéphale la parole exerce un puissant contröle social et, en ce sens, elle est, avec la violence, un des plus importants moyens de régulation.

\section{Le paradigme de la violence}

Ainsi que nous l'avons w, les combats réguliers peuvent être interprétés comme des procédures de type judiciaire et pénal dans lesquelles la compensation par rapport à l'offense ne s'effectue pas sous la forme d'une récupération (nourriture, biens, femmes par exemple) mais sous la forme d'une destruction opérée par les coups donnés (hématomes, blessures, fractures). En d'autres termes, la compensation pour l'offensé réside dans le fait que l'offenseur accepte de recevoir des coups et autorise, par là-mêrne, l'offensé à exprimer sa colère et sanctionner son agresseur. Pour ce dernier, elle réside dans la possibilité qui lui est offerte de rendre à son tour les coups reçus.

On peut sinterroger sur la nature de cette procédure qui octroie un droit de réciprocité à l'offenseur. Il semblerait en effet que seule une procédure intégrant la réciprocité de la destruction rende acceptable le combat aux yeux de ceux qui sont ainsi interpellés - c'est-àndire rende acceptable l'idée de se laisser volontairement frapper - et permette idéalement de mettre un terme à l'application du principe de rétorsion. L'échange de coups occupe une place intermédiaire dans l'ensemble des relations sociales et remplit une fonction de médiation qui s'oppose cependant à celle de la prise de parole et de l'échange de mots dans la vie quotidienne et lors des fêtes. Les combats se situent donc en-deçà de ce que l'on peut appeler l'échange positif (échange de biens, visites, discours, fêtes, nourriture, femmes), constitutif de la relation d'alliance, et participent de l'échange négatif (échange de mal, malêtre, maladie et de mort), constitutif de la relation dhostilité. Mais is jouent un röle médiateur dans les relations dhostilité car ils permettent de canaliser la violence de manière à ne pas rompre définitivement les relations d'alliance.

Pour leur part, les agressions occultes représentent une compensation par la destruction (mal, malêtre, maladie et mort) qui vise à éviter la contre-destruction. Elles permettent de temporiser dans la mesure où elles sont une stratégie pour ne pas provoquer l'ouverture dhostilités armées, mais elles créent et maintiennent la distance et l'évitement car elles alimentent la conception persécutive du malheur, de la maladie et de la mort, et, du mème coup, favorisent les réactions d'agressivité. 
Le flechage et thomicide volontaire, entin, sont les formes de violence les plus achevées en tant que procédures programmées d'extermination des parties. Au cours des combats réguliers la violence peut échapper au contröle a uquel elle s'est soumise, mais dans les raids guerriers elle est prémeditée et organisee. Cependant, l'exercice de la violence n'est pas systematiquement mis en cuvre : les vengeances peuvent péricliter car les Yanomami font des compromis par désir de tranquillité. Is peuvent avoir peur des lèches ou craindre un rapport de force défavorable quand le camp adverse comprend un ou plusieurs waitheri, hommes réputés particulièrement agressifs et combatifs, tandis qu'il n'y en a pas dans le leur. Les forces qui poussent dans le sens de l'hostilité sont aussi contrebalancées par celles agissant dans le sens de la paix exercée par la parenté et l'affinité. tinsi, des factions peuvent ne pas vouloir prendre parti pour l'un des deux camps car eiles comptent dans l'un et l'autre des parents et des alliés. Et, dans le cours des événements, tandis que certains hornmes influents exhortent à l'accomplissement des vengeances et à la guerre, d'autres, au contraire, interviennent pour demander la cessation des hostilites (wasii, dire de ne pas faire).

L'agression guerrière et la violence symbolique qui la sous-tend font de la mise à mort par fléchage le plus haut degré de violence. Toutefois, on n'accorde pas dans cet te société de valeur particulière au nombre d homicides perpétrés. L'exploit guerrier n'est pas spécialement exalté, fêté, chanté ou célébré, et aucun système de rang n'est associè à la pratique guerrière. Les guerriers n'acquièrent pas de titre de noblesse ni $n$ augmentent leur prestige en accumulant les victimes comme dans d'autres sociétés amérindiennes. I s'agit pour les Yanomami davantage d'un devoir à accomplir énvers leurs parents, leurs beaux-parents, les corésidents et les communautés alliées.

Limage du tueur n'est ni flatteuse ni flattée, mais la conduite waitheri réponù à une valeur essentielle de la société car le courage. la vaillance, comme l'agressivite déclaree et démontrée, permettent d'asseoir une réputation forçant au respect des droits, autrement dit, elles permettent d'inspirer la crainte du rapport de force et d'éviter ainsi les agressions.

On ne tue pas un autre Yanomami sans encourir soi-mème des risques extremes ${ }^{16}$ et mettre en péril la vie de ses parents et celle des membres de son groupe. Les raids pour venger les morts sont particulièrement associés aux fètes cérémonielles au cours desquelles sera consommée dans une bouillie de bananes ou bien versée dans le foyer une partie des cendres du corps incinéré. La vengeance a cours tant qu il reste des cendres. Certaines gourdes les contenant sont conservées suffisamment d'années pour que les fils du mort, une fois parvenus en àge, soient informés et accomplissent la vengeance. C'est le meurtrier qui doit payer préférentiellement et qui sera la cible privilégiée. Son nom est donné as mannequin de feuilles ligoté ( $n \dot{f} u w e \ddot{)})$ que l'on flèche avant une attaque, ce qui est une des conditions symboliques assurant le succès du fléchage réel, pratique ensuite. Mais un membre de sa parenté ou de son village peut lui étre substitue au nom du principe de la responsabilité collective.

Après tout homicide, effectue en solitaire ou à plusieurs, les meurtriers effectuent le rite unokaimou. Ce dernier est destiné à contrebalancer les effets nocifs 
du meurtre perpétré et à déjouer les rites d’agression intentés par l'intermédiaire du cadavre et qui doivent garantir la mort prochaine du ou des meurtriers. Dès l'instant où il a frappé, on peut considérer que le corps du meurtrier est limage en miroir du corps de la victime : le meurtrier ressent les effets de mal-ètre du corps de sa victime en train de succomber à ses blessures, tout comme postérieurement il ressent les effets de la décomposition et/ou de la crémation du cadavre ${ }^{17}$. C'est ainsi qu il est informé - sj personne ne rapporte des nouvelles de l'évolution de l'état de la victime. Sous linfluence du mort, le meurtrier toumenté perd son allant, sa vivacité et sa vigilance, aussi doit-il observer des régles trés strictes (notamment jeûner et éviter de se frotter ou de se gratter fortement et directement).

Le cadavre peut ètre suspendu plusieurs semaines dans des branchages ( $p e \ddot{k i}$ roomakei) pour quil se décompose et tombe en putréfaction avant qu'il ne soit procédè à son dépeçage et à la dislocation du squelette. Ces opérations ont pour but de retarder la crémation du corps du mort et de prolonger d'autant la durée du rite accompli parallelement par le meurtrier dont le corps réagit par analogie. Durant ce temps, à la mise en place de différents éléments et de différentes matières (comme des substances provoquant des démangeaisons) sur des parties spécifiques ou en rapport avec le corps du défunt, correspondent des réactions particulières du corps du meurtrier (comme des maux de ventre et des troubles digestifs amokumou et des ulcérations washëai consécutives à des lésions de grattage ${ }^{18}$ ). Le traitement du corps de défunt a pour objectif d'empècher le meurtrier de réussir l'épreuve et de sortir sans dommages de l'expérience. Les blessures, le refroidissement, la décomposition, l'écoulement du corps suspendu et la crémation sont ressentis par le meurtrier par un effet de dédoublement du corps du mort sur son propre corps. Lümage du corps pénètre en lui, lui donne des cauchemars et l'empéche d'accomplir le rituel unokaimou qui doit lui permettre d'éviter les effets délétères de cette influence somatisée. On peut penser que si le meurtrier ressent les changements d'état de sa victime c'est que le meurtre est vécu métaphoriquement comme une incorporation de la dépouille.

$\mathrm{Ne}$ pouvant, faute de place, traiter ici de l'ensemble des rites funéraires, des rites d'agression et de leur symbolique, pour comprendre le traitement rituel du meurtrier examinons le seul rite d'agression effectue la veille d'un raid waitherimou (avoir le comportement des êtres agressifs et violents). Au cours de ce rite. les hommes clament leur soif de vengeance, leur désir de tuer, leur $\alpha$ faim de viande, et ils naikinmou, déclarent quils sont naiki, quils vont faire une expédition de chasse, et promettent quils ne reviendront pas bredouilles... tu cours de ce rite encore, ils watupamou, font comme le vautour, le charognard. pratique qui symbolise la mise à nu des ossements. Les hommes watupamou avant l'exécution d'un raid, au retour d'un raid, ce qui annonce son succès, lors de la suspension du corps d'un individu mort par léchage ou par sorcellerie et lors du dépeçage de son corps, ce rite représentant dans chacun des cas la mise à mort d'un ennemi.

Les nombreuses métaphores associant le Yanomami (l'ètre humain) fléché et le gibier chassé montrent que la violence exercée consiste à rabajsser l'ennemi au 
rang animal. Mais si les activités de chasse sont ici confondues, le gibier humain se distingue implicitement du gibier chassé : de l'un ce sont les chairs crues et/ou faisandées qui sont consommées, de l'autre ce sont les chairs boucanées et/ou bouillies. On ne consomme d'ailleurs pas la viande d'un gibier qui a émis un bruit particulier (i. e. encore vive) quand on l'a découpee, ni de la viande boucanée qui a faisandé (i. e. pourrie) avant le retour des chasseurs à leur maison, car cela présage dans chacun des cas la mort prochaine d'un guerrier. Si le cannbalisme figuré des ennemis mis à mort par fléchage représente une violence extrême, c'est que le cannibalisme réel des parents et des proches alliés s'oppose bien distinctement au cannibalisme métaphorique des ennemis. D'un côté l'on consomme des 08 , de l'autre l'on consomme des chairs et des organes. Un corps est brülé, ses os sont broyés et réduits en cendres, ils sont bus mélangés à de la bouilie de bananes, la totalité de leur ingestion fait sur un temps long ; l'autre corps est dévoré sans aucune préparation dans l'immédiateté. Les o8 représentent la partie impérissable du corps et la continuité, les chairs et les organes la partie périssable et la discontinuité. L ingestion des uns correspond à la récupération de l'efficacité vitale du défunt, tandis que l'ingestion des autres correspond à sa destruction. On voit par là que, contraire de l'anthropophagie des parents et des proches alliés, le meurtre et le fléchage d'un Yanomami constituent sur le plan du reel et du symbolique les rapports extrêmes dhostilité et de distance culturelle.

Dans la société yanomami, le système d'agression est un élément constitutif de la structure sociale. La violence dont nous avons décrit les principales formes - combats, sorcellerie, guerre - qu'elles soient virtuelles ou réalisées, fonctionne comme un rapport de force dissuasif au service de la défense des droits privés et collectifs. La violation des droits et l'atteinte à l'intégrité corporelle des individus sont sanctionnées physiquement par des coups et par des morts et socialement par des fissions et des déplacements. On peut exprimer par le moyen d'un schéma les diffërentes formes de violence, patente ou occulte, correspondant aux différentes sphères de relations sociales et politiques :

Distance rela tive

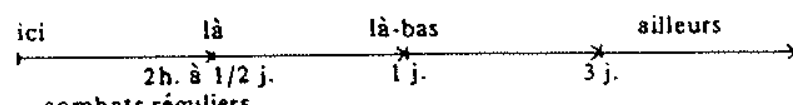

combats réguliers
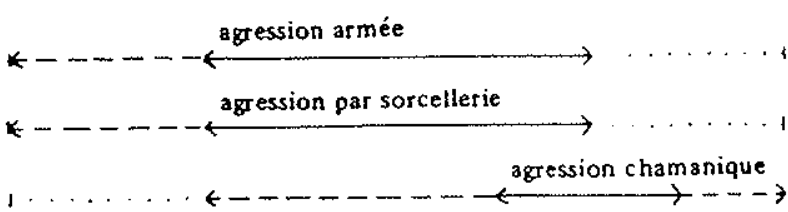

agression double-2nima

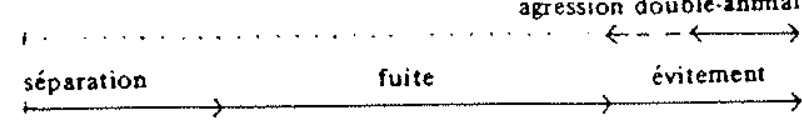

Relocalisation 
Dans cette logique, violence et guerre apparaiszent davantage comme une insti. tution garantissant l'existence et la reproduction d'une loi, soit d'un rapport symbolique unissant les différents groupes locaux entre eux.

Par rapport à ce système qui s'exerce comme force centrifuge, les relations de parenté et d'alliance matrimoniale jouent comme force centripète. Elles permettent le retablissement de relations pacifiques et empecchent l'exacerbation de la violence développée lors des conflits. Elles sont ainsi à la fois un facteur de rapprochement et de rassemblement en cas de désunion et un facteur de contention dans le choix et la pratique des modes de résolution des conflits. Mais ni le lien de consanguinité ni le lien d'affanité ne suffisent à éliminer tous les risques d'affrontement et de séparation. Cette impuissance empèche toute fixité dans l'espace, et la pérennisation dans le temps des groupes locaux, entraine le change. ment de localisation et de composition des groupes, et promeut la réalisation d'alliances matrimoniales en dehors de la proche affinité classificatoire.

Néanmoins, les pratiques de la violence peuvent intervenir de manière bien différente pour constituer l'ordre social.

Dans les rapports intra-et inter-communautaires, en l'absence de systèmes d'autorité constitués, elles produisent de l'ordre et permettent de répondre aux multiples tensions qui traversent la société.

Par contre, la violence exercée par un sexe sur l'autre reproduit un ordre préalable. L'autorité des hommes sur les femmes est perpétuée par un rapport de crainte basé sur un usage potentiel ou cffectif de la force. Cette violence sera le fait, au sein de la famille, du père et des frères de la femme, de son mari et de ses frères, voire des hommes de son groupe d'appartenance. Elle se pratique alors de manière unilatérale et fonctionne comme système pénal. Linégalité que l'on observe dans l'exercice de la violence entre les sexes correspond à une structure d'au torité et de pouvoir et permet de reproduire la domination masculine.

\section{NOTES}

1. Nous ne pouvons cependant le suivre lotejement, notamment en qui conceme linfanticide sélectif des nouveaux-nés de sexe féminin, le manque de fernmes te rapt des femmes et le type de liaison causale suggére entre ces différentes sortes de faits et la guerte. Vous détaillerons par ailleure ces points qui ne semblent pas correspondre avec les données que nous avons recueillies au centre de l'ethrie dans la Sierra Parima.

2. Le texte presenté ici sur la genèse de la guerre doit contribuer à montrer qu'elle ne saurat ètre déterminé par des contraintes d'ordre écologique comme la rareté des ressources animales ou des terres fertiles. Nous renvoyons à $N$. A. Chagnon [1968b] qui répond à A. Vayda [1961] lequel, à partir de deux modeles (les lban et les Maori) met en question les analyses de la guerte dans les socié tés des basses terres en Amerique du Sud, nolamment celles de J. Steward sur les Tupinamba et de R. Murphy sur les Mundurueu, et établit que les sociétés d'essarteurs en expansion font la guerre pour obtenir des territoires [vois encore

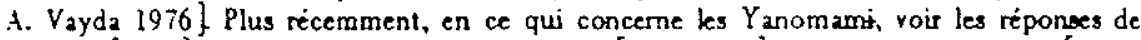
J. Lizot [1977] et de N. A. Chagnon et R. Hames [1979-1980] awx theses de M. Harris [1971, 1972, 1974, 1977, 1979] et de D. R. Gross [1975]

3. Les bandes sont plus nettement définies quand if y a eu fusion de deux ou plusieurs communautés. C'est sur la base de ce schema de division que se constituent en général en cas de conflit intracommunautaire, les parties adverses et que s'operent les fissions. 
4. Selon le critere yanomami, ces maisons sont à portée de voix, lorsqu'on crie.

5. Ces enserobles de populations ne sont pas totalement homogènes il y a possibilité de recrutement à l'intérieur d'un bloc de egments de population d'origine disférente et inversement, possibilité de alliement de certains de ses segments à d'autres blocs. C'est par rapport à ce cadre de référence que nous partons d'un groupe d'origine commune.

6. Nous ne voulons p2s dise que les femmes ne sont pas capables de combativite ou qu'elles n'interviennent ni ne participent d'aucune manière au système de l'agression.

7. Par exemple, le fait d'agresoer dit nopēmou (nopē : étranger/ennemi ; mou : avoir le comportement de) et devenir un ennemi napéprou (napē : étranger/ennemi ; prou : chargement d'état/se transformer en).

8. L'arme tenue dans Ia main peut etre encore un bois de cervidé un os d'animal (te fémur d'un jaguar par exemple) tailié en pointe, l'extrémité sectionnée d'un bois d'arc ou son équivalent fabriqué dans du bois de palmier.

9. Il existe une autre technique de combat (ekayou ou akrimou) que nous décrivons phas loin. Elte est pratiquée lors des fétes reafu à l'zide d'arnes tranchantes et précède généra. lement un combat patikai. Elle correspond à une forme exacerbee de violence par rapport à ce type de tutte.

10. Le bâton naprushi peut aussi étre épointé à la base pour ètre utilisé comme un épie u au cas oi $k$ combat toumerait mal. Nous n'avons pas eu connaissance de cas de batailles rangées à l'épieu qui représenteraient un degré aupérieur de violence par rapport à la bataille au napnsshi. Sa pratique semble ètre extremement rare (N. A. Chagnon en reporte un seut cas $[1968 \mathrm{~b}$ ou $1968 \mathrm{c}$ ], Les Yanomami disposent d'autres ames encore pour s'affronter comme la pique en bois de palmier ou en bambou. La pique est davantage employce à l'encontre des femmes ou des visiteurs lorsqu'on est en colère, pour menacer lorsqu'un combat dégénère, ou se defendre contre des incurseurs. En ce qui concerne le fusit, les Yanomarni n'ont pas encore, pour la phupart, accés à ce type d'arme. Pour la partie vénézuélienne les sources d'approvisionnement éventuelles sont limitées aux zones de contact avec les missions. Elles sont beaucoup phus nombreuses du còté brésilien et il amive que certains groupes brésiliens viennent aider un groupe vénézuében, amés d'un fusil.

11. Si, pris de fureur parce qu'il a trouvé son jardin pillé ou endommagé son chien blessé o tué, un homme $x$ précipite pour détruire un autre jardin ou tuer le chien du coupable présumé (il arrive fréquemment qu'il se trompe de cible), c'est lui qui devra ensuite baisser la tète le premier en quabité d'offenseur.

12. Soit quand son mari est reparti dans sa propre communauté après avoir accompli une periode de service marital (siyoframou), soit quand elle revient en visite chez elle apres avoir déjà vécu dans la communauté de son mari (même s'il l'accompagne, il est en position de faiblesse et $x$ fit rouer de coups), soit encore pendant une période où elle est retoumé vivre chez elle car enceinte ou fächée arec son mari.

13. Ces cas sont fréquemment imputés à l'utilisation de chames mortels, tenus dans le poing du combattant ou bien frottés sur la pierre, le casse-téte ou le bàton.

14. A propos de disputes ou de discordes interindividuelles, les Yanomami, hommes ou fernmes, hirai publiquement dans la maison commune. Cette parole, au ton particulierement azresuif, a pour but d'exprimer, immédiatement, en présence de l'offenseur le mécontentement et de denoncer des comportements et des attitudes irrespectueuses ou contraires a ses droits (insultes, moqueries, mensonges, prononciation du nom personnel d'un mort ou d'un adulte, vol ou endommagement de biens, de nourriture, de femmes).

15. Ces discours sont prononcés sur un ton stéréotypé, parlé ou crié, selor qu'ils sont pacifiques ou agressifs, et ils sont accompagnés d'une gestuelle formelle. Ils s'effectuent preférentiellement le sois ou la nuit quand tout le monde a rejoint son hamac, mais peuvent intervenir à tout moment de la joumce pourva que les intéressés soient présents. Ils ont un indice d'écoute et une durée qui varient en fonction du sujet ćvoqué, et de l'äge des orateurs, plusieurs hommes pouvant parler successivement. Les femmes d'un certain age peuvent aussi s'exprimer, parfois, en kanoamou. Les femmes en général en rajoutent (yëtêmou) sur ce qui est dit quand un mari ou un fils kanoamou sous une forme crice (hiroi). 
16. Chaque fois qu'un $Y$ anomami en the un au tre, il accomplit un rituel, y compris quand il tue un animal susceptibie d'ëtre le double d'un Yanornami. Mais dans le cas des agressions occulles, le meurtrier préfése cacher son forfait pour ne pas risquer d'ètre dénoncé, n'accormplissant done le rituel que s'il s'agit d'une victime d'une communaute étoignes. Si sa victime est trop proche, id ira pleurer le mort afin de ne pas etre découvert.

17. Quand on brîle le corps, les mains du meurtries $x$ couvrent de cloques des cendres sortent de sa bouche et de son nez. Quand on le suspend, be meurtrier $x$ courre d'une couche de crasse et des vers tombent de ses cheveux. Quand on depece le cadarre, il vomit et déglutit des cheveux, ce qui n'est pas sans éroquer la pelotte régurgité par le charognard.

18. De la meme maniete qu'on ne touche pas le sang d'un mort on ne louche pas un meurtrier accomplisesant le rite, pour éviter d'avoir des ulcérations.

\section{BIBLIOGRAPHIE}

Ales, C. et J. Chiappino

1981-82 Approche de la maladie et de ia therapie chananique chex les Yanomami (Venezuela), Les Cahiers de IORSTOM, série Sc. hum., vol. XVIII, no 4 : $110-125$.

1983 C.Medical add. Shamanism and Acculturation among the Yanomamis, m.s. (à paraitre dans The Health and the Suvivol of Veneruelion Yanoama. ARC) IR'GIA/SI Document 37).

Biocea, E.

1965 Yanoama. Récit d'une femme bréslienne enlevee por les Indiens. Paris, Plon.

Chagnon, N. A.

1966 Yonomamö Warfare, Social Organization and Marriage Alliances (Ph. thesis), Ann Arbor, University of Michigan.

1968 a The Culture-Ecology of Shifting (Pioneering) Cultivation among the $Y$ anomassö Indianss, Proceeding of the VIII International Congress of Anthropologicol and Ethnological Sciences, Tokyo, vol. $3: 249-255$.

$1968 \mathrm{~b}$ 'Yanomamō Social Organization and Wariares, in F'ar : the Anthropology of Armed Conflict and Aggression, M. Fried, M. Harris \& R. Murphy (eds.) Garden City-New York. Natural History Press : 109-159.

1968c Yonomomo : the Fierce People. New York, Holt, Rinehart \& Winston.

Chagnon. V. A.\& R. B. Hemes

1979 Protein Deficiency and Tribal Warfare in Amazoniz: New Datas, Science, $203: 910.913$.

1980 o $\mathrm{L}_{2}$ 'hipotesis proteica' y la adaptacion indigena a la cuenca del Ámazonas: una revision critica de las datos y de la teorias, Interciencia, vol. 5 (6) : 346-358.

Gross, D. R.

1975 Arotein Capture and Cultural Development in the Amazon Basins, American Anthropologist, $77: 526-549$.

Harris, M.

1971 Culture, Man, Nature : an Introduction to Ceneral Anthropology. New York, Thomas Y. Crowell. 
Harris, M.

I don't Know how you can Write Anything of Value if you don't Orfend Someones, Psychology Taday, $8: 6469$.

1974 Cows, Pigs, Wars and Hitches: the Riddles of Culture. New York, Random House.

1977 Sroteins and the Fierce Peopies, Cannibals and Kings: the Origin of Cultures New York, Random House.

1979 The $Y_{\text {anomamo }}$ and the Cause of $\mathrm{H}_{25}$ in Band and Village Societiess, in Braril, an Anthropological Perspective. Essays in Honor of Charles Wagley, M. Mangolis \& W. Carter (eds.). New York, Columbia University Press: 121.132 .

Lizot, 3 .

¿Population, ressources et guerre chez les Yanomamis, Libre, $2: 111.145$.

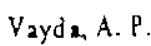

1961 Expansion and Warfare among the Swidden Agriculturists, American Anthropologitt, $63: 346.358$.

1976 War in an Ecological Perspective : Persistence, Change and Adaptatif Processes in Three Oceanion Societies. New York. Plenum Press. 